http://dx.doi.org/10.21611/qirt.1992.043

\title{
Inversion of thermal wave infrared images
}

\author{
by R.L. THOMAS, L.D. FAVRO, D.J. CROWTHER \\ and P.K. KUO (*)
}

(*) Department of Physics and Institute for Manufacturing Research, Wayne State University, Detroit, MI 48202, USA.

\begin{abstract}
Pulse-echo thermal wave imaging is becoming a very useful technique for nondestructive evaluation of materials. However, the blurring created by lateral heat diffusion remains as an important problem affecting the quality of the images. We describe here an inversion technique, based on the Born approximation, which permits the elimination of this blurring in the case of planar defects, such as delaminations, which are parallel to the surface. Tested on experimental images, this technique demonstrates that it is possible to make significant improvements in the quality of thermal images.
\end{abstract}

\section{Nomenclature}

$\begin{array}{ll}\mathcal{L}^{-1} & \text { Inverse Fourier transform } \\ \otimes & \text { Convolution operator } \\ \ell & \text { Defect depth }(\mathrm{m}) \\ t & \text { Time }(\mathrm{s}) \\ \alpha & \text { Thermal diffusivity }\left(\mathrm{m}^{2} \cdot \mathrm{s}^{-1}\right) \\ \mathrm{x}, \mathrm{y} & \text { Space variables } \\ u, v & \text { Fourier space variables (spatial frequency domain) } \\ \mathrm{i}(\mathrm{x}, \mathrm{y}) & \text { Experimental thermal wave contrast image } \\ h(x, y) & \text { Propagation function } \\ o(x, y) & \text { Defect shape function } \\ \mathrm{l}(u, v) & \text { Inverse Fourier transform of the contrast image } \\ H(u, v) & \text { Fourier transform of the propagation function } \\ O(u, v) & \text { Fourier transform of the defect shape function }\end{array}$

\section{Introduction}

Recently, infrared thermal wave imaging has become an important means for nondestructive evaluation. Non invasive and contactless, this technique provides a good alternative for determination of thermal properties or for detection and evaluation of defects. In particular, the "Pulse-Echo" technique represents a very rapid and efficient solution for testing the structural integrity of a material. The principle is simple: The discharge of powerful flash lamps provides a rapid heating of the front surface of the material, generating a thermal wave pulse. This wave propagates in the material and is reflected back towards the surface by any inhomogeneities in the sample. The temperature of the surface is monitored by means of an infrared video camera. A real 
lime image acquisition system is used to obtain an image of the sample surface temperature at a predetermined time. The time dependence of a defect-free region is then eliminated to obtain the thermal contrast. The resulting image is a representation of the scattered pulse from the subsurface defect [1]. However, because of the lateral thermal diffusion of the thermal wave reflected toward the surface, the image of the defect is blurred. The effect becomes more important when the defect is deep or when the acquisition time is long, and is such that a precise estimate of the size and shape of the defect is difficult. We demonstrate here a method which permits the recovery of the real shape of the defect. This method is based on a deconvolution of the infrared thermal wave echo image by a function which represents the attenuation and dispersion which took place throughout the propagation and reflection of the heat pulse. This type of technique is currently utilized in astronomy as well as in medical and optical imaging [2]. After an introduction to the model utilized here, we will present the algorithm used to invert our thermal wave images, then we will provide some examples of the inversion.

\section{Introduction to the model}

In the case of a planar defect parallel to the surface, located at a distance $l$ beneath the top surface, a Green's function solution to the heat equation has been carried out using the first order Born approximation and ignoring multiple scattering, giving us the following expression for the propagation function:

$$
h(x, y)=-\frac{A}{2 \pi}\left(\frac{1}{\pi \alpha t}\right)^{1 / 2} \frac{\partial}{\partial l} \frac{e^{-\frac{\left.\left[x^{2}+y^{2}+l^{2}\right]^{1 / 2}+l\right]^{2}}{4 \alpha t}}}{\left[x^{2}+y^{2}+l^{2}\right]^{1 / 2}}
$$

This propagation function completely describes the phenomena which take place in the thermal wave image formation: propagation and dispersion of the thermal waves, both in transit toward the scatterer and, following reflection, during the return of the pulse to the surface.

The following shape is assumed for the planar defect:

$$
\begin{array}{rlll}
O(x, y) & =1 & & \text { over the defect } \\
& =0 & & \text { elsewhere, }
\end{array}
$$

finally obtaining for the thermal contrast the expression:

$$
i(x, y)=\iint h\left(x-x^{\prime}, y-y^{\prime}\right) \cdot o\left(x^{\prime}, y^{\prime}\right) d x^{\prime} d y^{\prime}
$$

The general principle of the method is based on the fact that, as seen in Eq. (3), the thermal contrast image can be expressed as a convolution between the shape function and the propagation function: 


$$
i(x, y)=h(x, y) \otimes 0(x, y)
$$

or equivalently in Fourier space:

$$
I(u, v)=H(u, v) \cdot O(u, v)
$$

so that, since the form of the propagation function is known, from the experimental image $i(x, y)$ the shape of the defect can be obtained by:

$$
\mathcal{L}^{-1}[O(u, v)]=\mathcal{L}^{-1}\left[\frac{\mathrm{I}(u, v)}{H(u, v)}\right]
$$

\section{Inversion Algorithm}

The inversion procedure is as follows; the first step is to calculate the propagation function corresponding to the experimental case under investigation. It, along with the experimentally obtained contrast image, is transformed by means of a "Fast Fourier Transform (FFT)" algorithm [3]. The FFT of the thermal wave image is then divided by the FFT of the propagation function. An inverse FFT of the result of this division is then carried out to obtain the correct shape and size of the defect.

In fact it is necessary to carry out one intermediate step in this procedure. In Fourier space, the experimental image contains a considerable amount of high (spatial) frequency noise. Some of this noise originates in the detector, etc., but there is also noise which arises as a consequence of the 8-bit digitization of the signal by our imaging system. The result of this noise is that when the inverse FFT is performed (following division), the essential features of the defect in the image (in real space) are totally obscured. Consequently, it is necessary to filter out the high spatial frequencies prior to carrying out the inverse FFT. This filtering limits the resulting quality of the inversion, rounding off some of the sharp features in the image. In practice, we utilize a Wiener filter [4] and find that we are able to remove the high frequencies and still preserve the essential features of the size and shape of the defects of interest to us.

\section{Applications}

We have applied our inversion technique to two types of blurring which occurs in infrared thermal wave images: one arising from the depth of the defect and the other arising from the length of the acquisition time.

The first example is a specimen used to study the detection of second layer corrosion. It consists of two aluminum alloy plates, $2 \mathrm{~mm}$ in thickness. On the rear surface of one of the plates, three square $(38 \mathrm{~mm}$ on a side) regions of corrosion are simulated. Each region has had a different percentage of metal removed, corresponding to 25,50 and $75 \%$ simulated corrosion. As seen from the top surface, we thus have three identically shaped, known defects which differ only by their depths. Figure 1 shows the thermal wave contrast image of this specimen. This figure is a composite of three separate images, each of which was taken at a delay time following the flash heating such that the maximum contrast was obtained. Figure 2 shows the result of carrying out the inversion as described above. One can immediately see a better definition of the defect shapes, notably in the vicinity of the corners. The very weak signal from the deepest defect makes the inversion difficult in this case. However, even this inversion shows a definite improvement over the original image. 


\section{http://dx.doi.org/10.21611/qirt.1992.043}

The second example demonstrates the possibility of removing the blurring which occurs at long times. The specimen is a stainless steel plate, in the back surface of which has been machined a planar defect in the shape of a cross, $25 \mathrm{~mm}$ long overall. The defect is located at a depth of $1 \mathrm{~mm}$ beneath the top surface. We have imaged this defect at the following acquisition times following the heating pulse; $0.33,1.33$ and 2.33 seconds (Figs. 3, 4 and 5 ). One can see that the blurring has nearly obscured the shape of the cross at the longest acquisition time (Fig. 5), such that it appears more nearly to be a diamond shape. The results of our numerical inversions are shown in Figs. 6, 7 and 8, respectively. It is clear that the inverted images all show the same defect: the shape of the cross has been restored, even for the longest acquisition time case.

\section{Conclusion}

We have demonstrated that it is possible to carry out inversion of thermal wave images for which a large part of the blurring has been eliminated, thereby permitting a better description of the shape of the defect. The quality of the numerical inversion can be quite good, even for experimental images which contain high (spatial) frequency noise. It should be possible to improve the quality still further by increasing the dynamic range of digitization of the analog signal in the imaging system.

\section{References}

[1] P.K. Kuo, T. Ahmed, H.J. Jin, L.D. Favro, R.L. Thomas. - Synchronous thermal wave IR video imaging for NDE. J. of NDE, Vol. 8, No. 2, pp. 97-106, 1989.

[2] R.C. Gonzales, P. Wintz. - Digital image processing. $2^{\text {nd }}$ edition, Addison-Wesley Publishing Company, 1987.

[3] H.W. Press, B.P. Flannery, S.A. Teukolsky, W.T. Vetterling - Numerical recipes. Cambridge University Press, Cambridge, 1986.

[4] C.L. Fales, F.O. Huck, J.A. Cormick, S.K. Park. Wiener restoration of sampled image data: end to end analysis. J. Opt. Soc. Am., Vol. 5, No. 3, 1988.

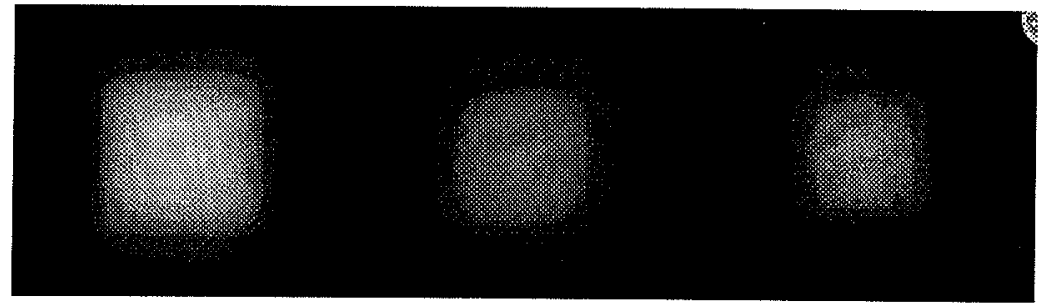

Fig. 1: Contrast images of defects representing 3 identically shaped corrosion regions, but a three different depths (see text). 
http://dx.doi.org/10.21611/qirt.1992.043

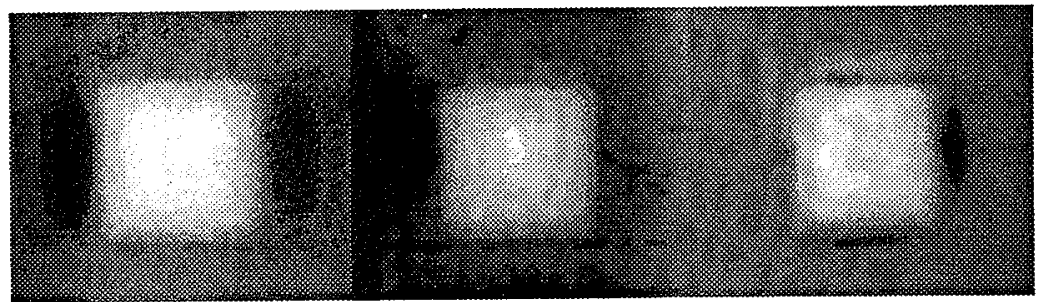

Fig. 2: Results of the inversion for the defects shown in Fig. 1.

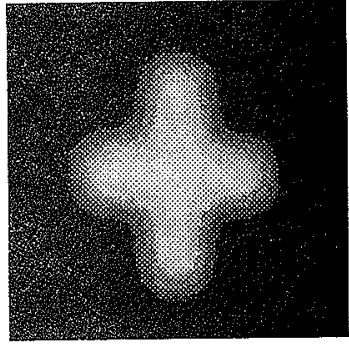

Fig. 3: Contrast image of the defect at $0.33 \mathrm{~s}$

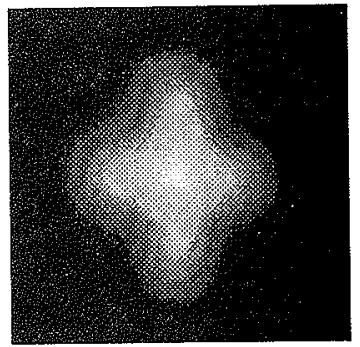

Fig. 4: Contrast image of the defect at 1.33s

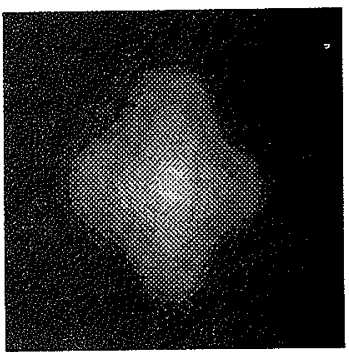

Fig. 5: Contrast image of the defect at 2.33s

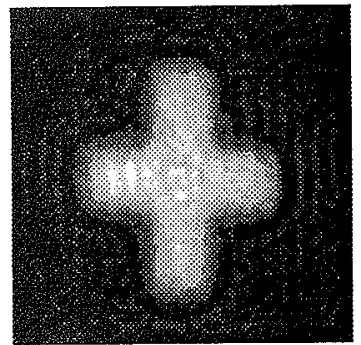

Fig. 6: Deconvolution result for the image of Fig. 3

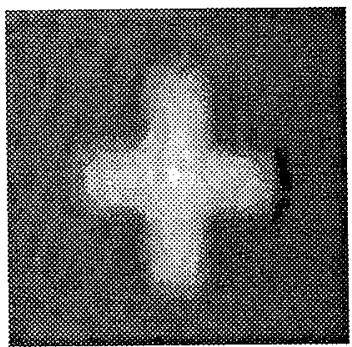

Fig. 7: Deconvolution result for the image of Fig. 4

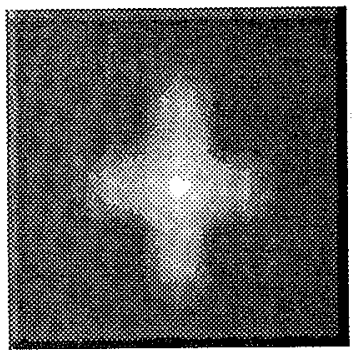

Fig. 8: Deconvolution result for the image of Fig. 5 